This article has been published in the Journal of Power Sources. The final publication is available at Elsevier via http://dx.doi.org/10.1016/j.jpowsour.2017.03.153

\title{
Characterization of Transport Phenomena in Porous Transport Layers using X-ray Microtomography
}

\author{
S. Hasanpour ${ }^{\mathrm{a}}$, M. Hoorfar ${ }^{\mathrm{a}}$, A.B. Phillion ${ }^{\mathrm{b}}$ \\ aSchool of Engineering, The University of British Columbia, Kelowna, BC, V1V1V7, Canada \\ ${ }^{\mathrm{b}}$ Department of Materials Science and Engineering, McMaster University, Hamilton, ON, L8S 4L7, Canada
}

\begin{abstract}
Among different methods available for the estimation of the transport properties of porous transport layers (PTLs) of polymer electrolyte membrane fuel cells, X-ray micro computed tomography $(\mathrm{X}-\mu \mathrm{CT})$ imaging in combination with image-based numerical simulation has been recognized as a viable tool. In this study, four commercially-available single-layer and dual-layer PTLs are analyzed using this method in order to compare and contrast transport properties between different PTLs, as well as the variability within a single sheet. Complete transport property datasets are created for each PTL. The simulation predictions indicate that PTLs with high porosity show considerable variability in permeability and effective diffusivity, while PTLs with low porosity do not. Furthermore, it is seen that the Tomadakis-Sotirchos (TS) analytical expressions for porous media match the image-based simulations when porosity is relatively low but predict higher permeability and effective diffusivity for porosity values greater than $80 \%$. Finally, the simulations show that cracks within MPL of dual-layer PTLs have a significant effect on the overall permeability and effective diffusivity of the PTLs. This must be considered when estimating the transport properties of dual-layer PTLs. These findings can be used to improve macro-scale models of product and reactant transport within fuel cells, and ultimately, fuel efficiency.
\end{abstract}

Keywords: X-ray microtomography; Porous Transport Layers; Permeability; Effective Diffusivity; Fuel Cell 


\section{Introduction}

The porous transport layer (PTL) is a critical component within polymer electrolyte membrane fuel cells (PEMFCs) as it facilitates fluid flow of the reactants and by-products, electron conductivity from the catalyst layer to the bipolar plates, heat conduction, and mechanical support for the membrane assembly [1]. PTLs, made mostly of carbon-based materials, can be classified as either single or dual layer. The single-layer PTL, consisting of carbon fibres and 5$30 \%$ PTFE loading, has a thickness between 150-500 $\mu \mathrm{m}$ and an effective pore size of approximately 10-30 $\mu \mathrm{m}$. The reasons for PTFE loading are to increase hydrophobicity of the carbon fibre mat and to bind the carbon fibres together. The dual-layer PTL contains an additional micro porous layer consisting of carbon particles mixed with PTFE that is coated onto the single-layer PTL. Within the literature, there is no convention on naming of sub-layers within the PTL. In this paper, the first layer (carbon fibre + PTFE) will be named GDL, while the second layer will be named MPL. Thus, a single-layer PTL consists of only GDL, while a duallayer PTL consists of both GDL and MPL.

The main properties related to transport phenomena [2] of PTLs include porosity, permeability, and effective diffusivity [3]. Porosity, $\epsilon$, is defined as the relative void volume within a material [4],

$\epsilon=1-\frac{V_{P T L}}{V_{\text {total }}}($ Eq. 1),

where $V_{P T L}$ is the volume of PTL material contained within a bounding volume $V_{\text {total }}$. Porosity is the main parameter of a porous medium, strongly correlating with all other properties of interest [5]. Permeability is defined as the structural resistance to a pressure driven flow. The wellestablished formula to define this characteristic is Darcy's law,

$-\nabla \mathrm{P}=\frac{\mu}{\mathrm{k}} \overrightarrow{\mathrm{v}}($ Eq. 2),

where $k$ is the absolute permeability of the porous medium, $\mu$ is the viscosity of the flow, $\vec{v}$ is the velocity of the fluid and $P$ is the pressure. Permeability is a structural property, and thus neither the viscosity, nor the applied pressure, nor the velocity affect its value. However, for Darcy's law to be valid, flow must occur in the low Reynolds number $(\operatorname{Re}<1)$ regime, as is the case in PEMFCs. Effective diffusivity, $f(\epsilon)$, is defined as the structural resistance against the movement of species from a region of high concentration to a region of low concentration [6], 
$f(\epsilon)=\frac{D_{o}}{D_{\text {eff }}}($ Eq. 3),

where $D_{0}$ is the bulk diffusion coefficient of a chemical species, and $D_{\text {eff }}$ is the apparent diffusion coefficient of a given porous medium. As PTLs are very thin in one direction as compared to the other two directions, these transport properties are anisotropic, i.e. having different values in the "in-plane" directions (across the thickness) and the "through-plane" direction (along the thickness).

$\mathrm{X}$-ray micro-computed tomography $(\mathrm{X}-\mu \mathrm{CT})$ is a non-destructive imaging technique that can provide the internal 3D structure of porous media. Recently, this method has been used extensively to quantify porosity in PTLs [4, 7-14]. A thorough literature review on this subject was provided in [10]. There are two main challenges when using X- $\mu \mathrm{CT}$ to quantify porosity in PTLs. The first challenge relates to binary segmentation, which is used to numerically separate PTL material from the background. Different thresholding techniques have been used in the literature [10, 13-15] to perform this task. Recently, Odaya et al. [13] showed that for high resolution 3D images of PTLs, a direct selection of a global threshold parameter is the most efficient while remaining quite accurate. The second challenge is identification of the bounding volume in which the material is enclosed. This difficulty is due to the fact that the surface of PTLs is quite rough. Hasanpour et al. [10] proposed a robust method to identify the surface of highly porous media, known as the Rolling Ball method, and demonstrated its applicability to PTLs.

$\mathrm{X}-\mu \mathrm{CT}$ has also been used to characterize transport phenomena in PTLs. In a study by Becker et al. [16], numerical methods were applied to the $3 \mathrm{D} X-\mu \mathrm{CT}$ images to quantify permeability, effective diffusivity, and electrical conductivity, which were then compared to experimental measurements. The effective diffusivity and permeability results showed very good agreement; however, the electrical conductivity results deviated from the experimental results, especially in the through-plane direction. Further, it was found that the through-plane effective diffusivity is lower than the in-plane value by a factor of two. Fishman et al. [17] used the porosity distribution data from $\mathrm{X}-\mu \mathrm{CT}$ in combination with an analytical model (the so-called TomadakisSotirchos [18] equations), to show the anisotropic nature of permeability and effective diffusivity within PTLs. Nabovati et al. [19] studied the effect of the heterogeneous porosity distribution on permeability of PTLs, concluding that higher through-plane heterogeneity leads to higher in- 
plane permeability. Instead of directly using the $\mathrm{X}-\mu \mathrm{CT}$ data to calculate permeability, this study used the porosity distribution data from $\mathrm{X}-\mu \mathrm{CT}$ to make a stochastic model with a simplified $3 \mathrm{D}$ structure. In another study, James et al. [20] performed X- $\mu$ CT on compressed PTLs to obtain $3 \mathrm{D}$ structures at $0 \%, 20 \%$ and $40 \%$ compression. The structures were then used as the input geometry for modelling diffusive flow, showing that the effective diffusivity of a sample decreases with increased compression. Furthermore, since higher compression leads to a decrease in porosity, samples with low porosity showed low effective diffusivity [20]. Although the use of X- $\mu$ CT to analyze transport phenomena in PTLs has shown to be quite effective, a systematic study comparing different PTLs has not yet been carried out. At present, the optimum size of the PTL representative volume element for simulating permeability and effective diffusivity is also unknown. A study conducted by Bernard et al. [21] on Al-Cu alloys showed that sample size plays an critical role when simulating transport phenomena in porous media using X- $\mu \mathrm{CT}$ datasets. In addition, in dual-layer PTLs it is known that the MPL has a significant effect on permeability and effective diffusivity. In general, the MPL contains lower overall porosity and very small pores (approximately $100 \mathrm{~nm}$ in size [22]), causing the permeability and effective diffusivity values to decrease by an order of magnitude [23] as compared to single-layer PTLs. However, the MPL also contains large cracks (formed during the coating process), which can be hundreds of microns in size [24]. The effect of cracks within the MPL on dual-layer PTL transport phenomena is unknown.

In this study, a comprehensive analysis of the transport properties (porosity, permeability, and effective diffusivity) of three commercially-available single-layer PTLs (SGL 35BA, SGL 35BC, Toray 090 and Freudenberg H2315 I6) is carried out using X- $\mu$ CT imaging and image-based numerical simulations. The effect of cracks within the MPL on the transport phenomena in duallayer PTLs is also examined. The research focused on both providing quantitative data for validation of analytical equations, and investigating property variation within the same sheet. Although the transport properties of PTLs has been previously investigated, to date there has not yet been a systematic study of multiple products from multiple vendors, nor a study on property variability. The results provide a complete dataset that can be used to inform macro-scale simulations of gas flow in PEMFCs, and to provide new recommendations for improving diffusion media. 


\section{Experimental Methodology}

\subsection{Materials}

Four commercially available PTLs were investigated, SGL 35BA and SGL 35BC (donated by SGL Carbon group, Wiesbaden, Germany), as well as Toray 090, and Freudenberg H2315 I6 (purchased from College Station, TX, United States). SGL 35BA is a single-layer PTL with 5 $\mathrm{wt} \%$ PTFE loading and a specified thickness of $300 \mu \mathrm{m}$. SGL $35 \mathrm{BC}$ is a dual-layer PTL with 5 $\mathrm{wt} \%$ PTFE loading and a specified thickness of $325 \mu \mathrm{m}$ [25]. The Toray 090 and Freudenberg H2315 I6 were selected due to their thickness and PTFE loading, which are similar to SGL 35BA, enabling a direct comparison between different manufacturing processes.

\subsection{Scanning Procedure}

The tomographic imaging was performed using a Zeiss microXCT-400 computed tomography microscope, following the procedure outlined in Odaya et al. [13]. Each sample was cut to a size of $4 \mathrm{~mm}^{2}$ in cross-section and firmly secured to the sample holder to minimize sample flutter during image acquisition. For each scan, 2500 radiographs were acquired spanning $360^{\circ}$ using the 20X objective with the image captured via a CCD camera. These radiographs were then used to reconstruct a $3 \mathrm{D}$ volume, $900 \times 900 \times 300$ voxels each with an edge length of $1.167 \mu \mathrm{m}$.

\subsection{Image Processing}

The ImageJ and Avizo software packages were used for image processing. After image acquisition, each 3D dataset was post processed to reduce noise and converted to a binary form. Further, in order to characterize the effect of MPL on the transport properties, it was necessary to segment the GDL and MPL from each other.

(a) PTL Binarization: For binarization, a median filter with kernel size of 2 was first applied to remove small artifacts [24], and smooth variations in radiograph quality. Then the data was binarized to separate the PTL from the background using a manual segmentation process whereby a single unique value is chosen as the threshold calue for the entire image [10].

(b) Segment MPL and GDL: For dual-layer PTLs, there was the additional requirement to segment the MPL from the GDL. Although the GDL and MPL can be visually distinguished, their greyscale values are relatively similar. Segmentation was achieved as follows. First, the dual-layer PTL was segmented from the background using global thresholding. Then, using 
hysteresis thresholding, the GDL was separated from the background. Finally, the MPL was segmented by subtracting the GDL from the dual-layer PTL. Note that $\mathrm{X}-\mu \mathrm{CT}$ can only resolve cracks within the MPL, not the very small pores, since they are below the resolution of the Zeiss microXCT-400 instrument.

\section{Numerical Methods used to Characterize Transport Phenomena}

\subsection{Porosity Estimation}

The main challenge in using $\mathrm{X}-\mu \mathrm{CT}$ to estimate porosity in PTLs is determination of the sample volume. Evaluation of the sample volume is strongly linked to the identification of the surface, since the sample is so thin. For this study, the surface identification method introduced in [10] is employed. In this method, the radius of the carbon fibre is used as the characteristic length, in combination with a distance transform function, to identify the PTL's surface topology from the segmented 3D dataset. Then porosity can be estimated following Eq. (1).

\subsection{Simulation of Permeability}

The permeability of a porous medium can be determined by simulating fluid flow within that medium, solving the Stokes equations and then inputting the relevant values into Darcy's law shown in Eq. (2). In order to find the permeability of PTLs, the Absolute Permeability Experiment Simulation tool within the Avizo Xlab module of the Avizo commercial software has been employed, using a convergence criterion of $10^{-6}[26]$

The main assumptions and the initial and boundary conditions within this software are as follows: (1) four planes parallel to the main flow direction are added to the simulation domain to act as walls preventing fluid loss; (2) two regions perpendicular to the main flow direction are added to the simulation domain to act as stabilization zones where the pressure is quasi-static; (3) the inlet and outlet pressure are given a value of 130 and $100 \mathrm{kPa}$ respectively, while the dynamic viscosity of the fluid is assumed to be 0.001 Pa.s; (4) the solid phase is impermeable. A schematic of the permeability simulation is shown in Figure 1(a). The two stabilization zones are placed on the top and bottom, while two of the four walls are visible and located on the sides. An example geometry and streamlines from a flow simulation can also be seen.

\subsection{Effective Diffusivity Determination}


The effective diffusivity is modelled using the Molecular Diffusivity Experiment Simulation tool within the Avizo Xlab module of the Avizo commercial software. To determine the apparent diffusion coefficient of the porous medium, two reservoirs having the same volume but different concentrations are located on each side of the segmented dataset, and allowed to equilibrate via Fick's second law. The volume of these reservoirs is defined as 10 times the void space volume within the porous medium, while the remaining four sides are sealed, allowing for no diffusion to

occur. The simulation is terminated once equilibrium is reached, i.e., when $\frac{\partial \mathrm{C}_{\text {in }}(\mathrm{t})}{\partial \mathrm{t}}=-\frac{\partial \mathrm{C}_{\text {out }}(\mathrm{t})}{\partial \mathrm{t}}$. Then, the effective diffusivity is calculated using Eq. (3) assuming $D_{\mathrm{o}}=1 \mathrm{~m}^{2} \mathrm{~s}^{-1}$. A schematic of the model is shown in Figure 1(b). For these simulations, the initial reservoir concentrations were set to $1711 \mathrm{~mol} \mathrm{~m}^{3}$ and 0 ; however, these initial values do not change the effective diffusivity results. The convergence criterion was assigned a value of $10^{-6}$, matching the value for the permeability simulation.

\subsection{Representative Volume Element Size}

Numerical simulations of material properties of porous media at the microscale require the selection of an appropriately-sized representative volume element [18]. Figure 2 shows the results of a series of simulations to characterize the effect of representative volume element size on transport phenomena in PTLs in both the through-plane and in-plane directions. The domain size was varied between 50 and $650 \mu \mathrm{m}$ in the in-plane directions, as shown in Figure 2(a), keeping the thickness constant at $\sim 300 \mu \mathrm{m}$. The bulk porosity, in-plane and through-plane permeability, and in-plane and through-plane effective diffusivity were then calculated in each domain. As can be seen in Figures 2(b-d), all three quantities are seen to vary significantly in the size range $50-350 \mu \mathrm{m}$. The minimum size for reliably estimating the bulk porosity is $400 \mu \mathrm{m}$. Above $350 \mu \mathrm{m}$, the through-plane permeability is seen to reach a value close to steady state. For the in-plane permeability, the required domain size is larger, i.e., $450 \mu \mathrm{m}$. Although variation still exists in this quantity, the range of the difference is within $\pm 5 \mu \mathrm{m} 2$, which is acceptable for such a highly porous sample. The results also indicate that the domain size does not significantly affect the predicted value of effective diffusivity, although the use of a sample with edge length greater than $400 \mu \mathrm{m}$ seems to provide more reliable results in the through-plane direction. Thus, to summarize, a relatively large representative volume element (on the order of $500 \mu \mathrm{m}$ in the edge length) is required to model transport properties in PTLs. 


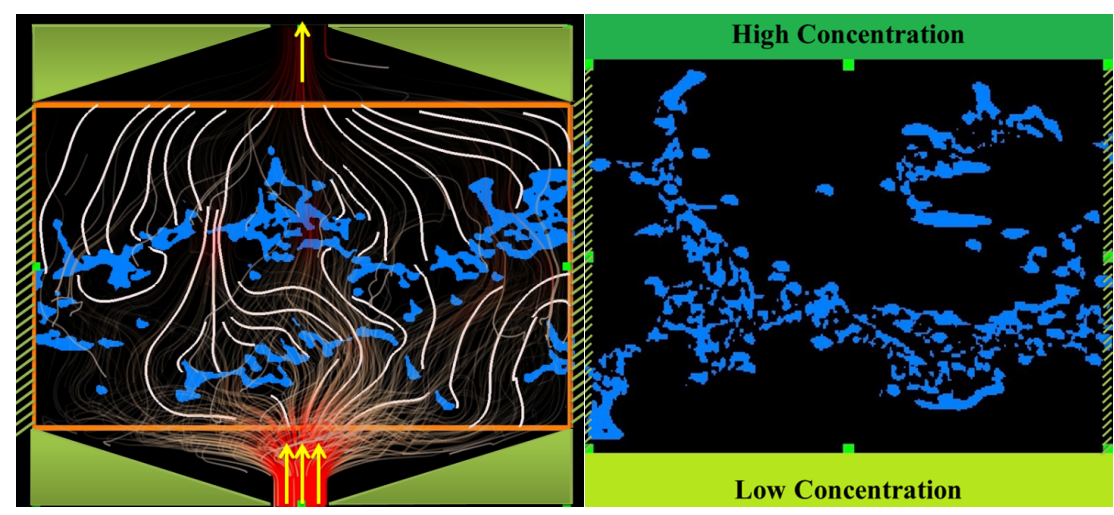

Figure 1: 2D Schematic showing the domain for the (a) permeability and (b) effective diffusivity simulations. The inner material is a cross-sectional slice of SGL 35BA.

\subsection{Determining the effects of MPL on Transport properties}

The effect of cracks within the MPL on the transport properties of PTLs can be determined by performing multiple Avizo Xlab simulations. For this analysis, first, the 3D image of a dual-layer PTL is used to find the through-plane permeability and effective diffusivity. Second, the same simulations were performed but on the individual, segmented layers. In dual-layer PTLs, the reactants and products are transported through cracks in the MPL. Cracks are located throughout this layer, and vary from $500 \mathrm{~nm}-100 \mu \mathrm{m}$ in length. Figure 3(a) shows an SEM image of the MPL surface, indicating the presence and frequency of surface defects within the MPL. X- $\mu C T$ imaging has the capability to extract some of the cracks (those that are larger than the resolution of imaging), as can be seen in Figures 3(b) and (c). This capability allows for a unique quantification of the effect of cracks on the transport properties of dual-layer PTLs.
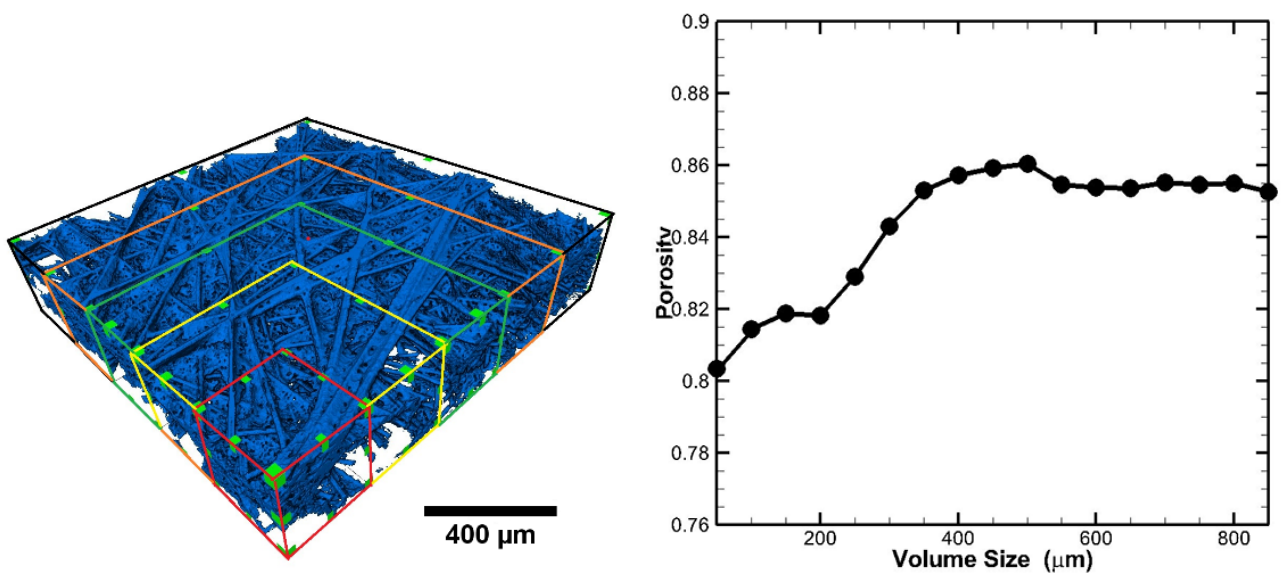


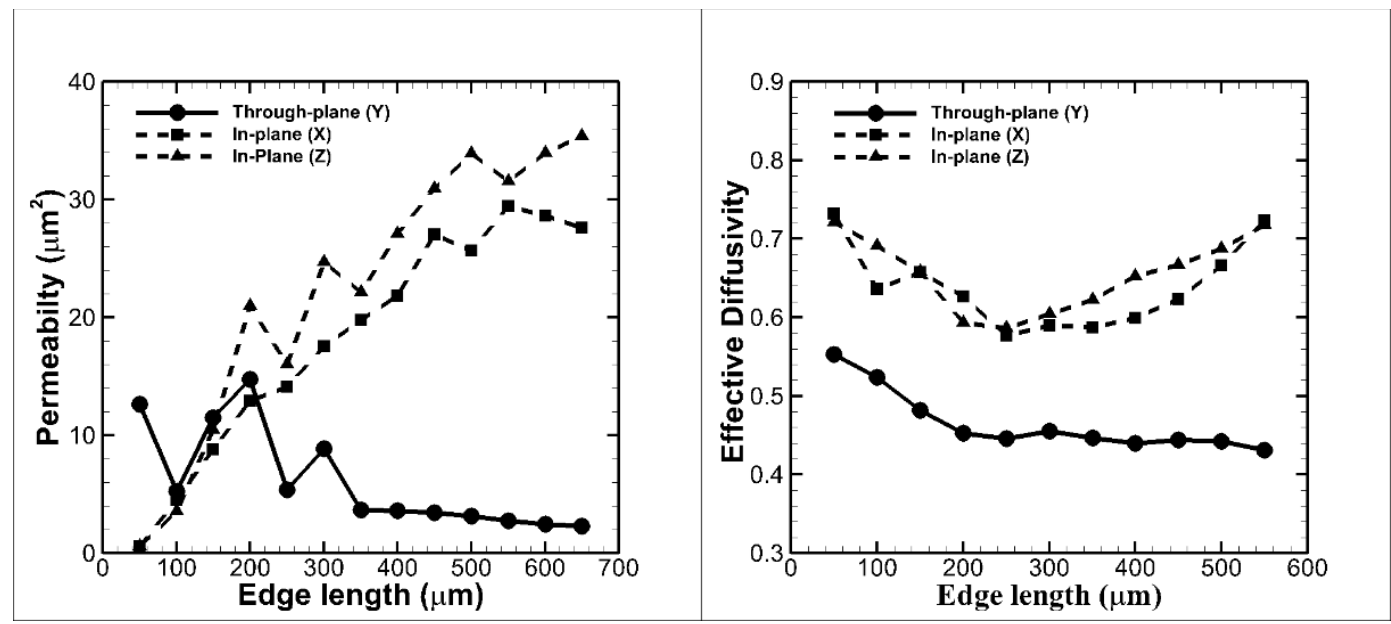

Figure 2: (a) 3D volume showing the different domains analyzed to determine the size of the representative volume element. Effect of domain size on the (b) bulk porosity, (c) simulated permeability, and (d) simulated effective diffusivity.

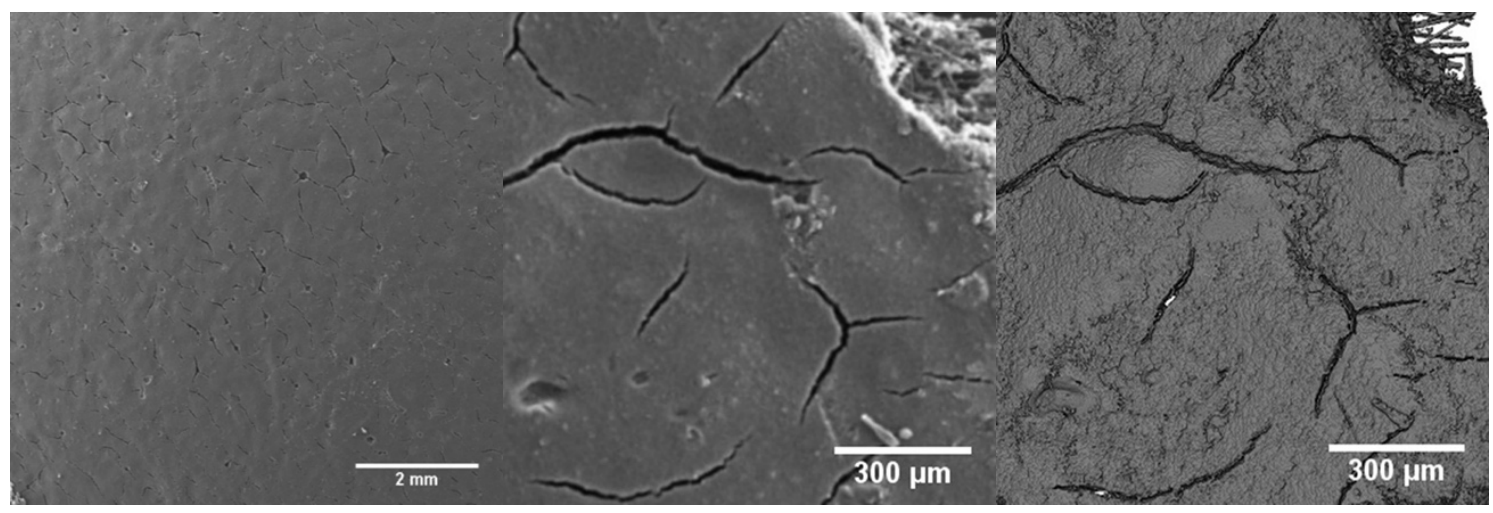

Figure 3:(a)SEM image of MPL(b)higher magnification and(c)X- $\mu \mathrm{CT}$ image of the same location of MPL

\section{Results and Discussion}

\subsection{D Structure}

Example denoised, binarized, and segmented 3D X- $\mu \mathrm{CT}$ images of each of the single-layer PTLs, along with the corresponding SEM images, are shown in Figure 4. In these 3D images, the random distribution of carbon fibres in the 3D structures can be clearly observed, along with the anisotropic distribution of the binder and PTFE. Further, the SGL 35BA is seen to be a quite open structure, whereas the Toray 090 and Freudenberg H2315 I6 appear to be significantly dense. The Freudenberg H2315 I6 also consists of curved carbon fibres, while the other samples have straight fibres. 


\subsection{Bulk Porosity}

The bulk porosity for the single-layer PTLs, and the GDL part of the dual-layer PTLs were characterized using the Rolling Ball method [10]. The carbon fibre radius for each PTL, used as the characteristic length, was determined from the scanning electron microscopy (SEM) images. Specifically, the average fibre radii were $5.36 \mu \mathrm{m}$ for SGL 35BA and SGL 35BC, $3.88 \mu \mathrm{m}$ for Toray 090, and $5.15 \mu \mathrm{m}$ for Freudenberg H2315 I6. The domain size for the porosity analysis was $900^{2} \mu \mathrm{m}^{2} \times$ sheet thickness.

\section{(a) Single Layer}

The bulk porosity was estimated on four locations from each PTL sheet (i.e., four X- $\mu \mathrm{CT}$ scans were acquired for each sheet). The results, i.e. the average bulk porosity and standard deviation, are summarized in Table 1. Highly porous PTLs, like SGL 35BA, show high variation in bulk porosity, while denser PTLs, like Freudenberg H2315 I6, show much smaller variation.

(b) Dual Layer

The bulk porosity of the GDL part of the dual-layer PTL SGL 35BC was calculated to be $82.6 \%$. SGL 35BC is a dual-layer version of SGL 35BA, and the fact that the two have similar porosity values (the difference between the average values of porosity of different samples of SGL 35BA and that obtained for the GDL part of SGL 35BC is 1.5 , which is within the standard deviation values obtained for these samples) indicates the validity of the segmentation method used to identify the GDL and MPL.

Table 1: Bulk porosity, permeability, effective diffusivity, and tortuosity for single-layer PTLs.

\begin{tabular}{|c|c|c|c|c|c|c|c|c|}
\hline \multirow{2}{*}{ Brand } & \multirow{2}{*}{$\begin{array}{c}\text { Bulk } \\
\text { Porosity }\end{array}$} & \multicolumn{3}{|c|}{ Permeability $\left[\mu m^{2}\right]$} & \multicolumn{2}{|c|}{ Effective Diffusivity } & \multirow{2}{*}{ Tortuosity } \\
\cline { 3 - 8 } & & TP & IP (1) & IP (2) & TP & IP (1) & IP (2) & \\
\hline \multirow{2}{*}{ SGL 35BA } & 84.2 & 7.9 & 59.8 & 46.7 & 0.68 & 0.82 & 0.82 & \multirow{2}{*}{$1.31 \pm 0.05$} \\
& \pm 2.5 & \pm 1.1 & \pm 30.5 & \pm 21.9 & \pm 0.03 & \pm 0.03 & \pm 0.03 & \\
\hline \multirow{2}{*}{ Today 090 } & 74.9 & 3.5 & 10.5 & 10.5 & 0.36 & 0.63 & 0.63 & \multirow{2}{*}{$2.11 \pm 0.07$} \\
& \pm 0.8 & \pm 0.4 & \pm 0.4 & \pm 2.3 & \pm 0.01 & \pm 0.02 & \pm 0.05 & \\
\hline Freudenberg & 69.4 & 1.9 & 5.7 & 5.2 & 0.49 & 0.62 & 0.60 & \multirow{2}{*}{$1.46 \pm 0.03$} \\
H2315 I6 & \pm 0.8 & \pm 0.4 & \pm 1.1 & \pm 0.8 & \pm 0.02 & \pm 0.03 & \pm 0.03 & \\
\hline
\end{tabular}




\section{SGL 35BA}
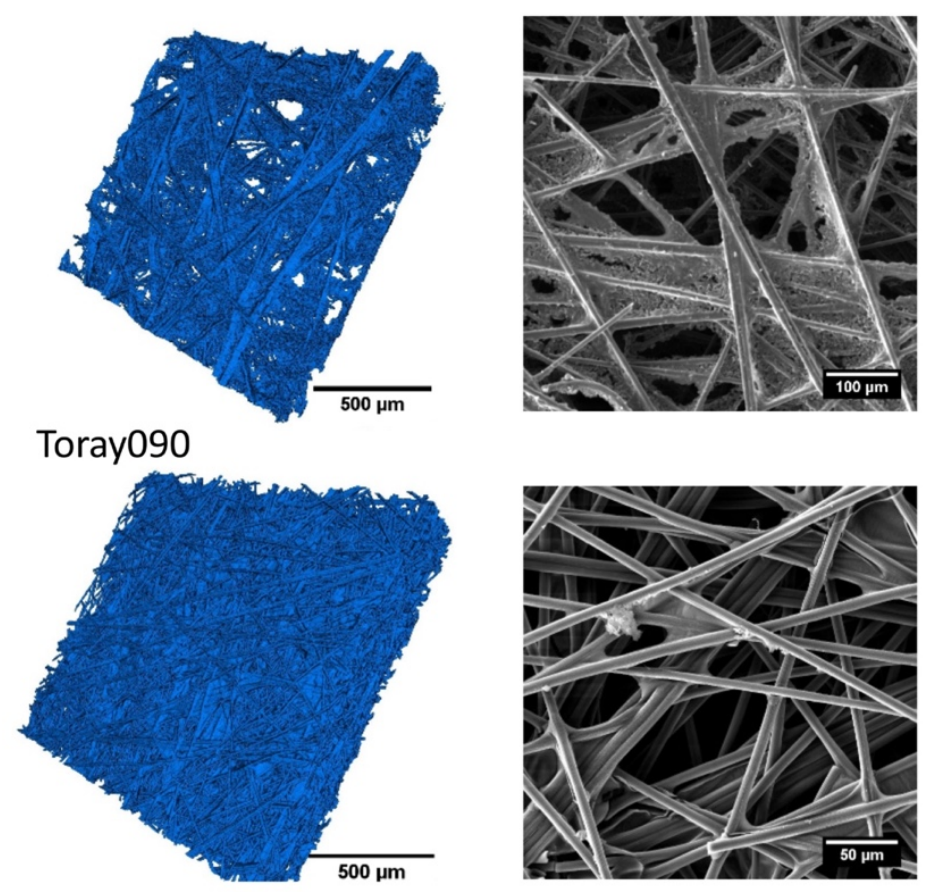

Freudenberg H2315 16
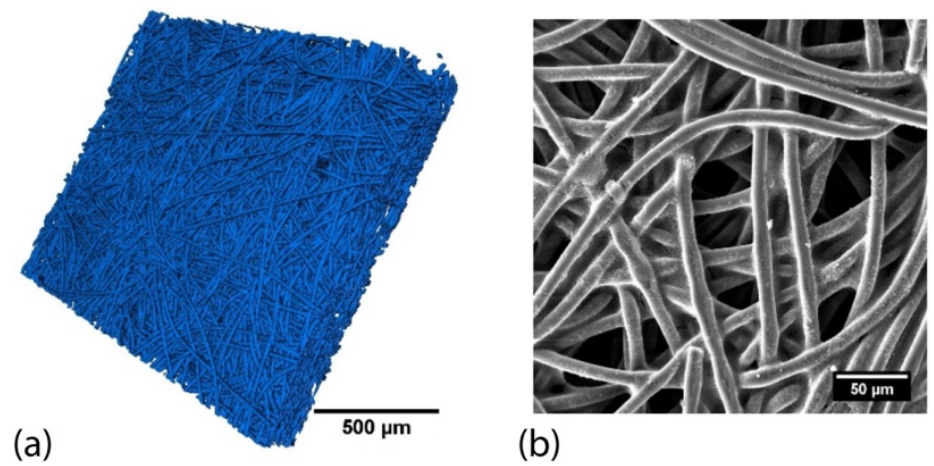

(b)

Figure 4: (a) 3D X- $\mu$ CT images and (b) 2D SEM images of SGL 35BA, Toray090, and Freudenberg H2315 I6.

\subsection{Permeability \\ (a) Single Layer}

The 3D X- $\mu$ CT images of the single-layer PTLs have been used to investigate the through-plane and in-plane permeability of these materials. Similar to the porosity assessment, the permeability analysis was carried out for four different samples of each PTL sheet. The domain size for the permeability analysis was $500^{2} \mu \mathrm{m}^{2} \times$ sheet thickness. The results are presented in Table 1 . The results show that the highly porous sample, SGL 35BA, has considerably higher permeability than Toray 090 and Freudemberg H2315 I6. Moreover, the through-plane permeability is 
approximately 2 to 10 times lower than the in-plane permeability, matching prior results reported in the literature [1]. Compared to SGL 35BA, Freudenberg H2315 I6 \& Toray 090 samples show more consistent results for both in-plane and through-plane permeability (the standard deviation for these two PTLs was one order of magnitude smaller than that obtained for SGL 35BA).

The data provided in Table 1 shows a significant variation in in-plane permeability of SGL 35BA between the different sheets, with a standard deviation of nearly $50 \%$. The reason for this enormous variation in permeability is related to the porosity; SGL 35BA has the highest overall porosity and the highest variation in porosity between samples. As shown in Eq. 4 (which will be discussed below), the relationship between porosity and permeability is nonlinear and at high values of porosity small changes in porosity will result in large changes in permeability. Structurally, the PTL is becoming quite open allowing for multiple pathways for fluid flow. A well-known analytical relation that predicts the permeability of fibrous materials is the Tomadakis-Sotirchos (TS) model [15]:

$k=\frac{\epsilon}{8(\ln \epsilon)^{2}} \frac{\left(\epsilon-\epsilon_{\mathrm{p}}\right)^{\alpha+2} \mathrm{r}_{\mathrm{f}}^{2}}{\left(1-\epsilon_{\mathrm{p}}\right)^{\alpha}\left[(\alpha+1) \epsilon-\epsilon_{\mathrm{p}}\right]^{2}}$

where $r_{f}$ is the fibre radius, $\epsilon$ is the porosity, and $\alpha$ and $\epsilon_{p}$ are material constants. The porosity $\epsilon$ is determined from the X-ray tomography images, although it must be calculated in the region of interest, which is different from the porosity values shown in Table 1 since they represent the entire sheet. The constants $\alpha$ and $\epsilon_{p}$ depend on the fibre distribution and orientation, and the direction of flow. For PTL materials, the fibres are distributed along the plane of the sheet. Under these conditions, the constants for the estimation of in-plane permeability, assuming that the flow direction is parallel to the fibres, are $\alpha=0.521$ and $\epsilon_{\mathrm{p}}=0.11$. Conversely, the constants for the through-plane permeability estimation, assuming the flow to be perpendicular to the fibres, are $\alpha=0.785$ and $\epsilon_{\mathrm{p}}=0.11$.

Figure 5 compares the permeability estimates calculated using the TS model and those obtained from the $\mathrm{X}-\mu \mathrm{CT}$ simulations for all three PTLs. In this figure, the symbols represent the simulation predictions, while the lines represent the TS-calculated permeability values. Three different lines are used, as each PTL sheet has a different fibre radius. The results for the inplane direction are given in Figure 5(a). As it can be seen, the simulation calculations and the TS model predict similar permeability values for samples with lower porosity, i.e., Toray 090 and 
Freudenberg H2315 I6. However, the simulation results deviate from the TS model for SGL 35BA having higher porosity. The results for the through-plane permeability are given in Figure 5(b). Similar to the in-plane permeability, the simulation and TS model results match at lower porosity, but there is a disagreement at higher porosity. Based on these observations, it appears that the TS model is useful in predicting permeability when porosity is relatively low (below $80 \%$ in these PTLs), but overestimates the permeability for high porosity samples. This is because the open nature of highly-porous samples increases the permeability variation, making the simulation much more structure-dependent.

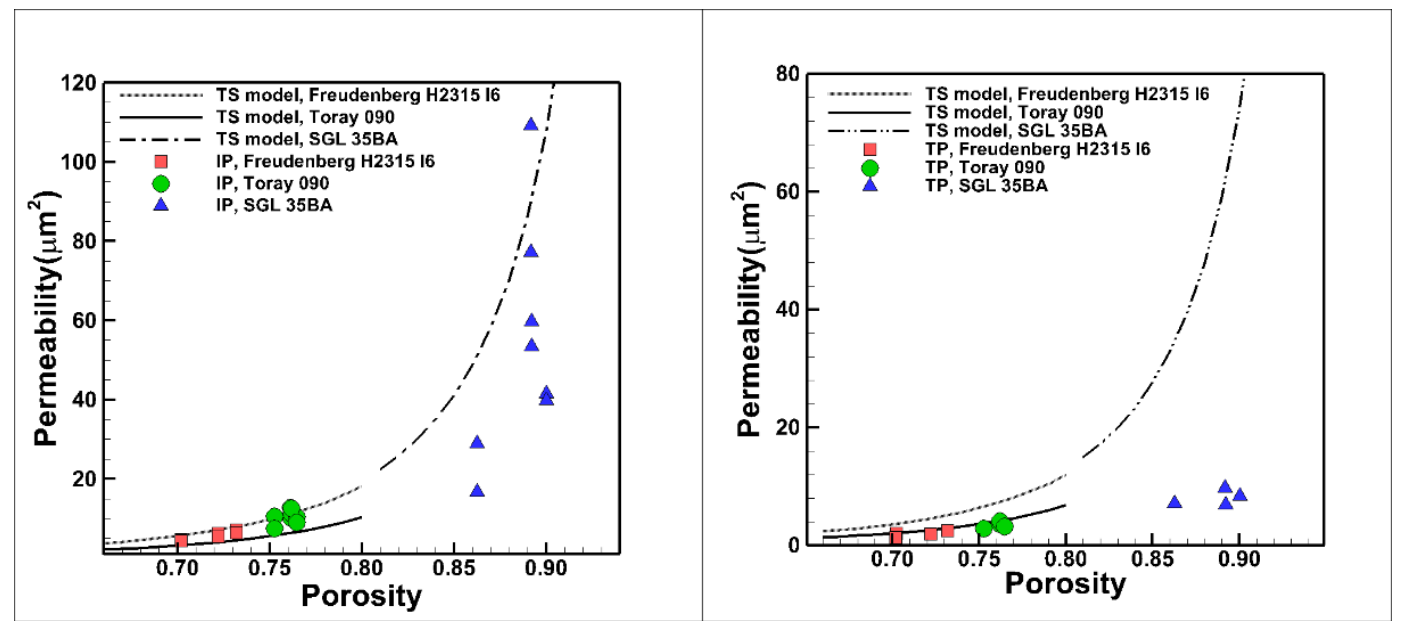

Figure 5: Comparison between permeability as calculated from the 3D X- $\mu \mathrm{CT}$-based simulations and estimates from the TS formula; (a) in-plane and (b) through-plane.

\section{(b) Dual Layer}

The MPL cracks play a significant role on the transport properties of the PTLs. Figure 6 shows the through-plane porosity distribution of SGL 35BC. The porosity value starts decreasing on the MPL (right) side of the graph. Without considering the nano pores found within MPL, since X$\mu \mathrm{CT}$ cannot resolve them, the $20 \%$ porosity in this layer is due solely to the cracks.

The 3D X- $\mu \mathrm{CT}$ image of the dual-layer SGL 35BC is shown in Figure 7(a). It consists of an open-cellular GDL with a fibrous structure and a thick MPL with cracks. The volume fraction of the GDL and MPL were 0.54 and 0.46. Images (b), (c), and (d) show the fluid stream-lines obtained from the through-plane simulations of each layer individually (b, c), and then when both are combined (d). As it can be seen, the flow is only able to pass through the cracks on the 
surface of the MPL in a limited manner (Figure 7(c)). Thus, fluid movement is hindered within the PTL due to the presence of the MPL, and hence permeability decreases for the dual layer. The quantified permeability values show that the large cracks, more than $1 \mu \mathrm{m}$, provide a major contribution towards the permeability of dual-layer PTLs. Specifically, the through-plane permeability of the GDL part was $16.4 \mu \mathrm{m}^{2}$, for the cracks within MPL it was $2.7 \mu \mathrm{m}^{2}$, and for the entire dual-layer structure it was $0.9 \mu \mathrm{m}^{2}$. These results are repeated in Table 2. Thus, the permeability of the dual-layer PTLs is an order of magnitude smaller than the permeability of the GDL part. These results are in general agreement with those reported in [20].

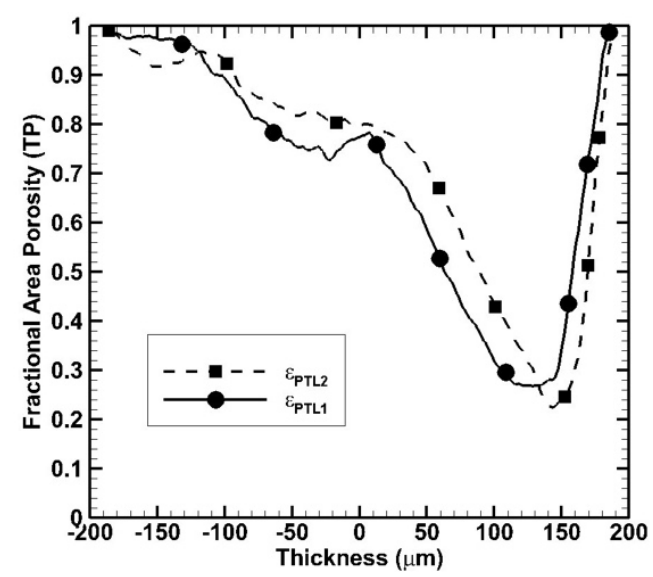

Figure 6:Variation the in area porosity through the thickness of the sample for dual-layer SGL $35 \mathrm{BC}$
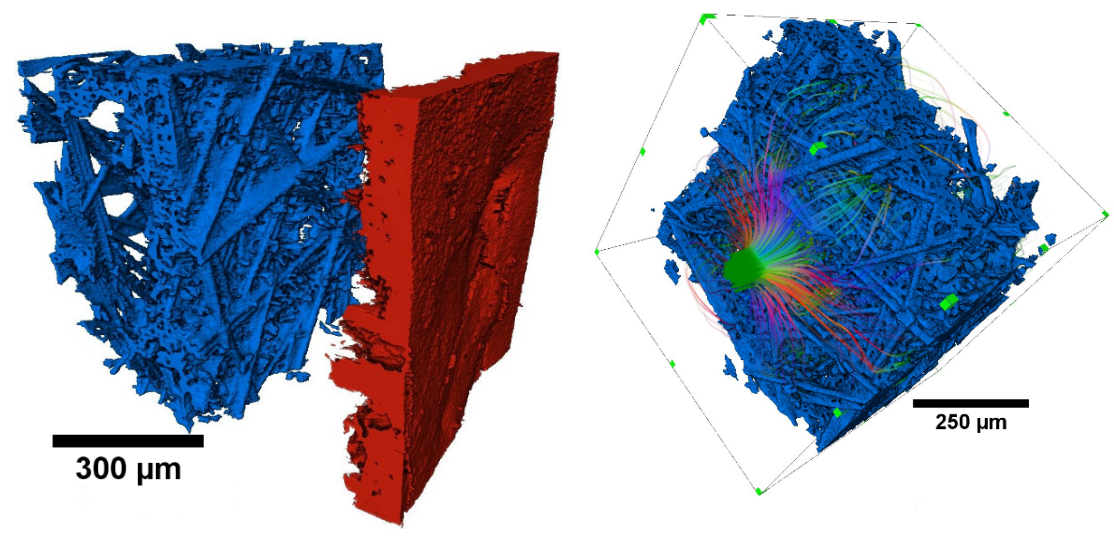

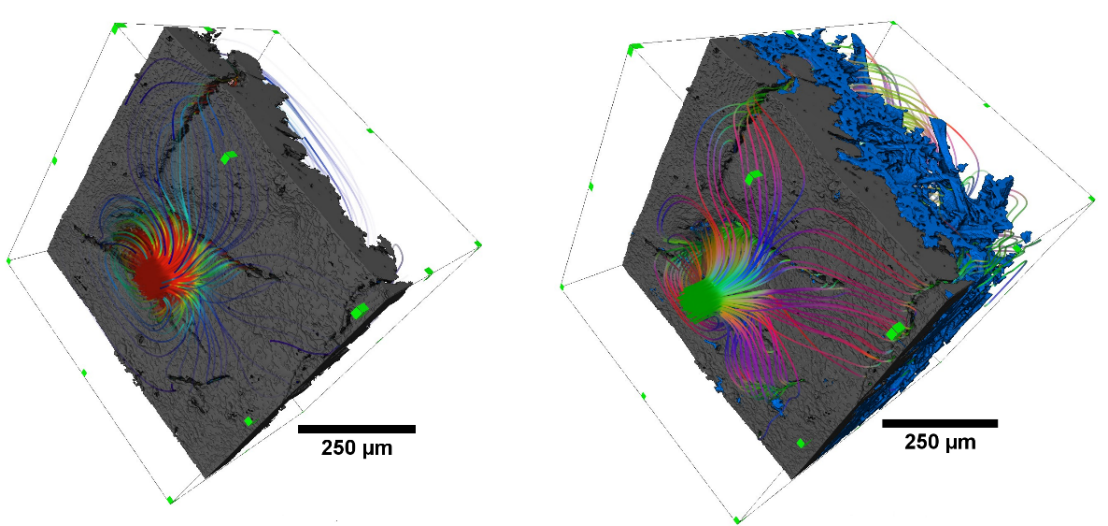

Figure 7: Dual-layer PTL SGL 35BC. 3D segmentation (a) showing the GDL (blue) and MPL (red) parts. Fluid streamlines from the (b) GDL-only simulation, (c) MPL-only simulation and (d) dual-layer simulation.

\subsection{Effective diffusivity}

(a) Single Layer

The in-plane and through-plane effective diffusivity was simulated on four different locations of each PTL sheet using the same domain as the permeability simulations. The results are summarized in Table 1, and shown in Figure 8, and also compared against the relevant Tomadakis-Sotirchos (TS) model:

$\mathrm{f}(\epsilon)=\epsilon\left(\frac{\epsilon-\epsilon_{\mathrm{p}}}{1-\epsilon_{\mathrm{p}}}\right)^{\alpha}$

where $\alpha$ and $\epsilon_{p}$ are the same material constants as used in Eq. 4. Beginning with the simulation results, it can be seen that there is relatively little variability in the predicted effective diffusivity within a single-layer PTL, and further, the in-plane effective diffusivity is similar to the throughplane results (although it is more variable). The in-plane variability could be due to the small thickness which may not provide enough cross-section to compute this value. Figure 2(c) also showed variability in the predicted in-plane effective diffusivity as a function of the edge length. Interestingly, the Toray 090, single-layer PTL, has a lower diffusivity as compared to the Freudenberg H2315 I6 PTL even though its porosity is higher. This discrepancy has also been reported before [17], and indicates that porosity is not the only parameter that must be considered when determining the effective diffusivity of PTLs. The other parameter that might have an 
effect on the effective diffusivity is the tortuosity of the medium, which can also greatly modify the effective diffusivity of samples having the same porosity value.

In comparing the results from the TS model and the simulation, Figure 8, it can be seen that there is good agreement in the in-plane direction. However, there is poor agreement in the throughplane direction as the TS model overestimates the effective diffusivity for a wide range of porosity values. Further, for Toray 090, the TS model prediction is significantly different. Thus, Toray 090 sample cannot be approximated using random-based analytical models. One possible source of error is that Eq. 5 does not include the effect of the tortuosity on the porous structure. The effective diffusivity can be used to determine the tortuosity of a PTL. Tortuosity, $\tau$, represents the ratio of the mean effective path length and the thickness of the sample [28], and can be used to estimate the time needed for reactants and products to enter or leave the PTL. The relation between the effective diffusivity and tortuosity is given by [29]:

$f(\epsilon)=\frac{D_{\text {eff }}}{D_{\mathrm{o}}}=\frac{\epsilon}{\tau}$

The tortuosity values in the through-plane direction for the three single-layer PTLs are listed in Table 1. These values are in agreement with prior work [30].

(b) Dual Layer

Table 2 summarizes the quantified effective diffusivity results based on the X- $\mu \mathrm{CT}$ based simulations in the dual-layer PTL SGL 35BC. The result of the GDL part $(f(\epsilon)=0.55)$ is similar to that of the corresponding single-layer PTL $(f(\epsilon)=0.68)$. But, the effective diffusivity of the large cracks within the MPL $(f(\epsilon)=0.17)$ was found to be three times less than that of the GDL, and the combined value $(f(\epsilon)=0.10)$ is even smaller. These results show that the cracks within MPL have a significant effect on species diffusion within PTLs. 


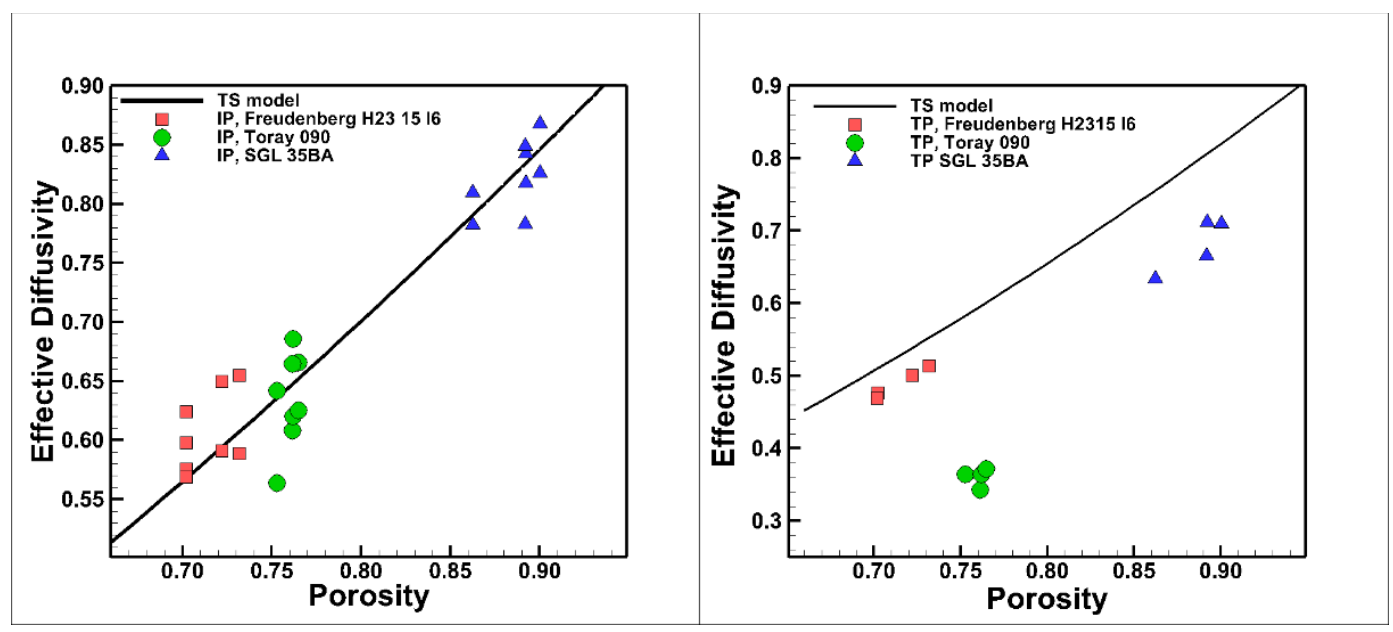

Figure 8: Comparison between effective diffusivity as calculated from the 3D X- $\mu$ CT-based simulations and estimates from the TS formula; (a) in-plane and (b) through-plane.

Table 2: Permeability and diffusivity predictions in dual-layer PTLs

\begin{tabular}{|c|c|c|}
\hline Layer & $\begin{array}{c}\text { Through-plane } \\
\text { Permeability }\left[\mu \mathrm{m}^{2}\right]\end{array}$ & $\begin{array}{c}\text { Through plane } \\
\text { Effective Diffusivity }\end{array}$ \\
\hline GDL & 16.4 & 0.55 \\
\hline MPL & 2.7 & 0.17 \\
\hline PTL & 0.9 & 0.10 \\
\hline
\end{tabular}

\section{Conclusions}

In this study, the transport properties of three porous transport layers used in hydrogen-based fuel cells has been determined using high resolution X-ray micro-computed tomography (X$\mu \mathrm{CT})$. The porosity of these PTLs were estimated using a newly developed method known as the Rolling Ball method [10]. Then, using Avizo Xlab simulation tools, the permeability and effective diffusivity of PTLs was obtained directly from the 3D images. To perform these simulations, a systematic study was undertaken to determine the ideal size of the representative volume element, in which the transport properties were independent of the sample volume.

The simulation results provided new insight into the variability of permeability and effective diffusivity both between PTL products, and within an individual PTL sheet. (1) The more porous PTLs (e.g. SGL 35BA), show much a larger variation in transport properties as compared to 
dense PTLs (e.g., Toray 090). (2) PTLs with increased porosity showed increased in-plane and through-plane permeability and effective diffusivity. (3) In-plane permeability and effective diffusivity are higher as compared to through-plane permeability and effective diffusivity for almost all twelve samples analyzed here. These results provide a complete dataset that can be used to inform macro-scale simulations of gas flow in PEMFCs, and to provide new recommendations for improving diffusion media.

The simulated transport properties were then compared against the results of the well-known Tomadakis-Sotirchos (TS) model. It was shown that the TS model overestimates the throughplane properties of samples with high porosity, especially for the through-plane permeability. Finally, the effect of cracks larger than $1 \mu \mathrm{m}$ in diameter within the micro-porous layer (MPL) of the dual-layer PTL on the transport properties of the porous material was investigated. The results showed that permeability and effective diffusivity of a dual-layer PTL is an order of magnitude less than those of a single-layer, and that flow through the MPL is entirely due to surface cracks on this layer. Thus, cracks play an important pathway for reacant and product flow through PTLs.

\section{Acknowledgements}

The authors thank the Natural Sciences and Engineering Research Council of Canada for funding this research, and the Canada Foundation for Innovation for funding the purchase of the X-ray tomographic microscope.

\section{References}

[1] M. Mathias, J. Roth, J. Fleming, W. Lehnert, Handbook of fuel cells. (2003).

[2] L. Cindrella, A. Kannan, J. Lin, K. Saminathan, Y. Ho, C. Lin, J. Wertz, J.Power Sources. 194 (2009) 146-160.

[3] Z. Fishman, J. Hinebaugh, A. Bazylak, J.Electrochem.Soc. 157 (2010) B1643-B1650.

[4] J.Z. Fishman, Investigation of Surface Properties and Heterogeneity in Gas Diffusion Layers for Polymer Electrolyte Membrane Fuel Cells. (2010).

[5] J.T. Gostick, M.W. Fowler, M.A. Ioannidis, M.D. Pritzker, Y.M. Volfkovich, A. Sakars, J.Power Sources. 156 (2006) 375-387.

[6] M. Ismail, D. Ingham, K. Hughes, L. Ma, M. Pourkashanian, Int J Hydrogen Energy. 40 (2015) 10994-11010.

[7] P.K. Sinha, P. Halleck, C. Wang, Electrochemical and Solid-State Letters. 9 (2006) A344A348. 
[8] F.N. Büchi, R. Flückiger, D. Tehlar, F. Marone, M. Stampanoni, ECS Transactions. 16 (2008) 587-592.

[9] R. Flückiger, F. Marone, M. Stampanoni, A. Wokaun, F.N. Büchi, Electrochim.Acta. 56 (2011) 2254-2262.

[10] S. Hasanpour, M. Hoorfar, A. Phillion, Electrochim.Acta. 185 (2015) 34-39.

[11] S. Hasanpour, A. Phillion, M. Hoorfar, (2015) V001T04A001-V001T04A001.

[12] S. Prass, S. Hasanpour, P.K. Sow, A.B. Phillion, W. Mérida, J.Power Sources. 319 (2016) 82-89.

[13] S. Odaya, R. Phillips, Y. Sharma, J. Bellerive, A. Phillion, M. Hoorfar, Electrochim.Acta. (2014).

[14] H. Ostadi, K. Jiang, P. Prewett, Micro \& Nano Letters, IET. 3 (2008) 106-109.

[15] N. Otsu, Automatica. 11 (1975) 23-27.

[16] J. Becker, R. Flückiger, M. Reum, F.N. Büchi, F. Marone, M. Stampanoni, J.Electrochem.Soc. 156 (2009) B1175-B1181.

[17] Z. Fishman, A. Bazylak, J.Electrochem.Soc. 158 (2011) B247-B252.

[18] M.M. Tomadakis, S.V. Sotirchos, J.Chem.Phys. 98 (1993) 616-626.

[19] A. Nabovati, J. Hinebaugh, A. Bazylak, C.H. Amon, J.Power Sources. 248 (2014) 83-90.

[20] J. James, H. Choi, J. Pharoah, Int J Hydrogen Energy. 37 (2012) 18216-18230.

[21] D. Bernard, Ø. Nielsen, L. Salvo, P. Cloetens, Materials Science and Engineering: A. 392 (2005) 112-120.

[22] H. Ostadi, P. Rama, Y. Liu, R. Chen, X. Zhang, K. Jiang, J.Membr.Sci. 351 (2010) 69-74.

[23] J. Pharoah, J.Power Sources. 144 (2005) 77-82.

[24] Z. Fishman, A. Bazylak, J.Electrochem.Soc. 158 (2011) B846-B851.

[25] Sigracet gdl $34 \& 35$ series gas di_usion layer, the gdl series speci_cation, . URL http://fuelcellsetc.com/store/DS/SGL-GDL_34-35.pdf.

[26] Avizo9.0.1. Module: Median filter (avizo lite).

[28] R. Thiedmann, C. Hartnig, I. Manke, V. Schmidt, W. Lehnert, J.Electrochem.Soc. 156 (2009) B1339-B1347

[29] Z. Fishman, A. Bazylak, J.Electrochem.Soc. 158 (2011) B846-B851.

[30] R. Flückiger, S.A. Freunberger, D. Kramer, A. Wokaun, G.G. Scherer, F.N. Büchi, Electrochim.Acta. 54 (2008) 551-559. 